\title{
Movement Imitation via an Abstract Trajectory Representation in Dorsal Premotor Cortex
}

\author{
(DAaron L. Wong, ${ }^{1 \star}$ Steven A. Jax, ${ }^{1 \star}$ Louisa L. Smith, ${ }^{1}$ Laurel J. Buxbaum, ${ }^{1}$ and John W. Krakauer ${ }^{2,3}$ \\ ${ }^{1}$ Moss Rehabilitation Research Institute, Elkins Park, Pennsylvania 19027, ${ }^{2}$ Department of Neurology, and ${ }^{3}$ Department of Neuroscience, Johns Hopkins \\ University School of Medicine, Baltimore, Maryland 21205
}

Humans are particularly good at copying novel and meaningless gestures. The mechanistic and anatomical basis for this specialized imitation ability remains largely unknown. One idea is that imitation occurs by matching body configurations. Here we propose an alternative route to imitation that depends on a body-independent representation of the trajectory path of the end-effector. We studied a group of patients with strokes in the left frontoparietal cortices. We found that they were equally impaired at imitating movement trajectories using the ipsilesional limb (i.e., the nonparetic side) that were cued either by an actor using their whole arm or just by a cursor, suggesting that body configuration information is not always critical for imitation and that a representation of abstract trajectory shape may suffice. In addition, imitation ability was uncorrelated to the ability to identify the trajectory shape, suggesting that imitation deficits were unlikely to arise from perceptual impairments. Finally, a lesion-symptom mapping analysis found that imitation deficits were associated with lesions in left dorsal premotor but not parietal cortex. Together, these findings suggest a novel body-independent route to imitation that relies on the ability to plan abstract movement trajectories within dorsal premotor cortex.

Key words: action planning; apraxia; imitation; motor planning; movement trajectories; stroke

\section{Significance Statement}

The ability to imitate is critical for rapidly learning to produce new gestures and actions, but how the brain translates observed movements into motor commands is poorly understood. Examining the ability of patients with strokes affecting the left hemisphere revealed that meaningless gestures can be imitated by succinctly representing only the motion of the hand in space, rather than the posture of the entire arm. Moreover, performance deficits correlated with lesions in dorsal premotor cortex, an area not previously associated with impaired imitation of arm postures. These findings thus describe a novel route to imitation that may also be impaired in some patients with apraxia.

\section{Introduction}

Imitation is critical for communicating through gesture, using tools, and acquiring new motor skills. The study of imitation has revealed that humans, unlike all other animals including nonhuman primates, are uniquely adept at copying meaningless gestures that have no evident goal or desired outcome other than the

Received Oct. 8, 2018; revised Jan. 9, 2019; accepted Feb. 19, 2019.

Author contributions: A.L.W., S.A.J., L.J.B., and J.W.K. designed research; A.L.W., S.A.J., and L.L.S. analyzed data; A.L.W. and S.A.J. wrote the first draft of the paper; A.L.W., S.A.J., L.J.B., and J.W.K. edited the paper; A.L.W. wrote the paper; S.A.J. and L.L.S. performed research.

This work was supported by National Science Foundation Grant BCS-1358756 to J.W.K., and National Institutes of Health Grant R01-NS065049 to L.J.B. We thank H. Branch Coslett for assistance with lesion identification; Stephanie Shaia and Harrison Stoll for assistance with the SVR-LSM analyses; and Peter Turkeltaub and Andrew DeMarco for sharing their SVR-LSM software.

The authors declare no competing financial interests.

${ }^{*}$ A.L.W. and S.A.J. contributed equally to this work as co-first authors.

Correspondence should be addressed to Aaron L. Wong at wongaaro@einstein.edu.

https://doi.org/10.1523/JNEUROSCI.2597-18.2019

Copyright $\odot 2019$ the authors action itself (Whiten et al., 2004; Subiaul, 2016). The processes that comprise this ability remain poorly understood.

Current theories of imitation largely stem from research into ideomotor apraxia. In this intriguing condition, caused primarily by a left-hemisphere lesion, patients can reach and grasp normally with their nonparetic ipsilesional arm (Buxbaum et al., 2005; Ietswaart et al., 2006) but cannot imitate (Buxbaum et al., 2014) with either arm. This prior work has suggested that humans imitate by matching the body configuration of the actor (Goldenberg, 2009, 2013). This theory, however, has primarily arisen from studying the imitation of static postures (Goldenberg, 1999) rather than dynamic gestures.

Imitating moving gestures becomes computationally challenging if a description of the changing body configuration is required, as it would require keeping track of the motion of each limb segment. A simpler approach would be to represent a gesture as the path traced by the end-effector (i.e., a movement trajectory). For example, when writing the letter "S," it is sufficient to specify only the sinuous shape along which the end- 
effector moves, rather than a sequence of arm configurations. Indeed, such abstract trajectory-path representations can explain kinematic invariance in handwriting across effectors (Wright, 1990; Rijntjes et al., 1999) or size scales (van Galen and Teulings, 1983; Wing, 2000; Kadmon Harpaz et al., 2014). We recently demonstrated that a trajectory-path representation is also used to plan reaches around obstacles (Wong et al., 2016). Moreover, this may explain how nonprimates, such as dolphins, imitate human actions despite lacking multisegmented arms (Taylor and Saayman, 1973; Bauer and Johnson, 1994). We therefore hypothesize a second route to imitation: one that does not specify body configurations, but is instead trajectorybased and body-independent.

If there are two routes to imitation, it is reasonable to ask whether these routes are functionally and anatomically dissociable. Current evidence suggests that imitation is associated with neural activity in the left inferior parietal and premotor cortices (i.e., Brodmann areas 39 and 40, and 6) (Molenberghs et al., 2009; Caspers et al., 2010; Buxbaum et al., 2014). However, a division of the dorsal stream into a dorsodorsal pathway concerned with reaching to spatially defined goals and ventrodorsal pathway concerned with body-based planning (Rizzolatti and Matelli, 2003; Binkofski and Buxbaum, 2013) suggests a neural architecture that could support two distinct routes to imitation: one that is body-based and one that is body-independent. Notably, neural activity in dorsal premotor cortex (PMd) has been associated with planning movement paths (Hocherman and Wise, 1990, 1991; Rijntjes et al., 1999; Pearce and Moran, 2012), and lesions in PMd impair planning reaches around obstacles (with reaches instead directed through the barrier) (Moll and Kuypers, 1977). Thus, we hypothesized that if there is a route to imitation involving the representation of a movement trajectory, the neural substrate of such a representation is in PMd rather than the inferior parietal lobule (IPL).

To address our behavioral and anatomical hypotheses, we asked neurotypical controls and patients with left hemisphere strokes to imitate meaningless movement trajectories. Imitation was tested using the left hand, corresponding in patients to the ipsilesional (unaffected) limb. Critically, participants were cued by watching either a cursor (providing no body-configuration information) or an actor. We reasoned that, if patients were equally impaired at imitating both stimuli, this would support a body-independent route to imitation. Patients had heterogeneous lesion locations that, as a group, included PMd and IPL, with the anticipation that patients would exhibit a range of imitation abilities depending on lesion size and location. This enabled us to test where in the brain movement-trajectory paths might be computed.

\section{Materials and Methods}

Twenty-two right-handed chronic stroke survivors with lesions confined to the left hemisphere ( 8 women and 14 men) and 13 right-handed neurologically intact controls (7 women and 6 men) completed the experiment. One patient was subsequently removed from the study before analysis due to impaired visual abilities. The remaining group of patients (Table 1; lesions depicted in Fig. 1) was marginally younger than the control group (patients: $57.4 \pm 10.1$ years, range $35-80$ years; controls: $64.2 \pm 11.1$ years, range $44-80$ years; $p=0.086$ ); thus, any detrimental effects of aging on performance would be reflected more heavily in the control group than the patient group. All participants provided written consent to participate in the study in accordance with the guidelines of the Einstein Healthcare Network Institutional Review Board and were compensated for their participation.
Table 1. List of patient participants with left-hemisphere strokes

\begin{tabular}{|c|c|c|c|c|c|c|}
\hline Patient & $\begin{array}{l}\text { Age } \\
\text { (yr) }\end{array}$ & Sex & $\begin{array}{l}\text { Time }(\mathrm{mo}) \\
\text { after stroke }\end{array}$ & $\begin{array}{l}\text { Lesion } \\
\text { volume }\left(\mathrm{mm}^{3}\right)\end{array}$ & $\begin{array}{l}\text { Western } \\
\text { Aphasia } \\
\text { Battery }^{a}\end{array}$ & $\begin{array}{l}\text { Meaningless } \\
\text { imitation }^{b}\end{array}$ \\
\hline P1 & 55 & $\mathrm{~F}$ & 176 & 151,318 & 9.4 & 0.75 \\
\hline P2 & 80 & M & 153 & 65,376 & 9.9 & 0.50 \\
\hline P3 & 64 & $M$ & 120 & 71,750 & 8.5 & 0.70 \\
\hline P4 & 37 & $\mathrm{~F}$ & 62 & 63,924 & 8.9 & 0.48 \\
\hline P5 & 72 & $\mathrm{~F}$ & 33 & 133,321 & 9.4 & 0.58 \\
\hline P6 & 55 & M & 172 & 50,976 & 10 & 0.78 \\
\hline P7 & 59 & M & 89 & 57,638 & 8.9 & 0.75 \\
\hline P8 & 65 & $M$ & 37 & 46,433 & 9.6 & 0.80 \\
\hline P9 & 64 & $\mathrm{~F}$ & 57 & 8793 & 10 & 0.78 \\
\hline P10 & 71 & M & 62 & 67,164 & 4.7 & 0.65 \\
\hline P11 & 35 & $M$ & 53 & 88,170 & 7.4 & 0.68 \\
\hline P12 & 52 & $M$ & 44 & 92,970 & 9.8 & 0.83 \\
\hline P13 & 51 & $M$ & 28 & 16,930 & 9.5 & 0.83 \\
\hline P14 & 56 & $\mathrm{~F}$ & 66 & 29,508 & 9.8 & 0.85 \\
\hline P15 & 57 & $M$ & 115 & 145,449 & 10 & 0.85 \\
\hline P16 & 49 & $\mathrm{~F}$ & 147 & 48,490 & 9.3 & 0.85 \\
\hline P17 & 55 & $M$ & 69 & 68,799 & 9.8 & 0.88 \\
\hline P18 & 43 & $\mathrm{~F}$ & 39 & 117,890 & 6.9 & 0.73 \\
\hline P19 & 59 & M & 52 & 135,780 & 8.5 & 0.63 \\
\hline P20 & 58 & $M$ & 94 & 17,710 & 10 & 0.95 \\
\hline P21 & 69 & $\mathrm{~F}$ & 22 & 30,450 & 10 & 0.78 \\
\hline
\end{tabular}

${ }^{a}$ The auditory comprehension subsection of the Western Aphasia Battery is scored out of a maximum of 10 points. ${ }^{b}$ This test involves the imitation of 10 meaningless gestures, scored according to qualitative accuracy of hand posture, arm posture, movement amplitude, and timing. Scores are average proportion correct. This is part of a larger apraxia battery.

All participants completed two tasks as part of this experiment: the Production task and the Perception task (details below). All tasks involved a set of trajectory shapes that were chosen such that the resulting gestures would be relatively arbitrary and did not have a commonly associated meaning. (Although it is possible to use a heuristic to link these gestures to meanings, such a strategy could be applied to any potential gesture selected for this task; hence, our selection criterion was simply that the entire spatiotemporal trajectory of the movement had no obvious meaning.) Each task consisted of two trial phases. The first phase was a practice block in which participants were provided with instructions on how to complete the task, were shown how to perform the task, and then allowed to rehearse three example trials in which they were given feedback regarding their performance. Following this, participants completed a test phase during which they received no further reminders of task instructions or feedback regarding their accuracy in performing the task. Participants received different stimuli during the practice and test blocks (test stimuli depicted in Fig. 2).

Trajectory Production task. In the Production task, participants executed 2D movements by sliding their fingertip along the surface of a vertically mounted sheet of clear Plexiglas. The left arm was used for all participants so that the stroke group would produce movements with the ipsilesional limb. Stimulus trajectories varied in size, but all were contained within a $56 \mathrm{~cm} \times 38 \mathrm{~cm}$ area at the center of the board. Participants sat with their hand resting in their lap (i.e., the "home" position) $\sim 50 \mathrm{~cm}$ from the Plexiglas, a distance that allowed them to comfortably perform movements. Instructions about the movements to perform were presented via a laptop computer placed $\sim 30 \mathrm{~cm}$ behind the Plexiglas. Each video clip, which ranged in length from 4.5 to $6.4 \mathrm{~s}$, was played twice with a $1 \mathrm{~s}$ delay between clips. Following the second video, there was a $1 \mathrm{~s}$ delay before a tone sounded to inform participants they should begin their movement. The movement consisted of reaching out and upward to contact the Plexiglas surface, generating the required trajectory in the vertical plane by sliding the hand along the Plexiglas, and then returning the hand to the home position in their lap. Participants were given the instructions to "use you left finger to trace the same path the dot or person made. Make sure your path looks exactly like the video's. It's very important that you be as precise as possible." If participants began to 


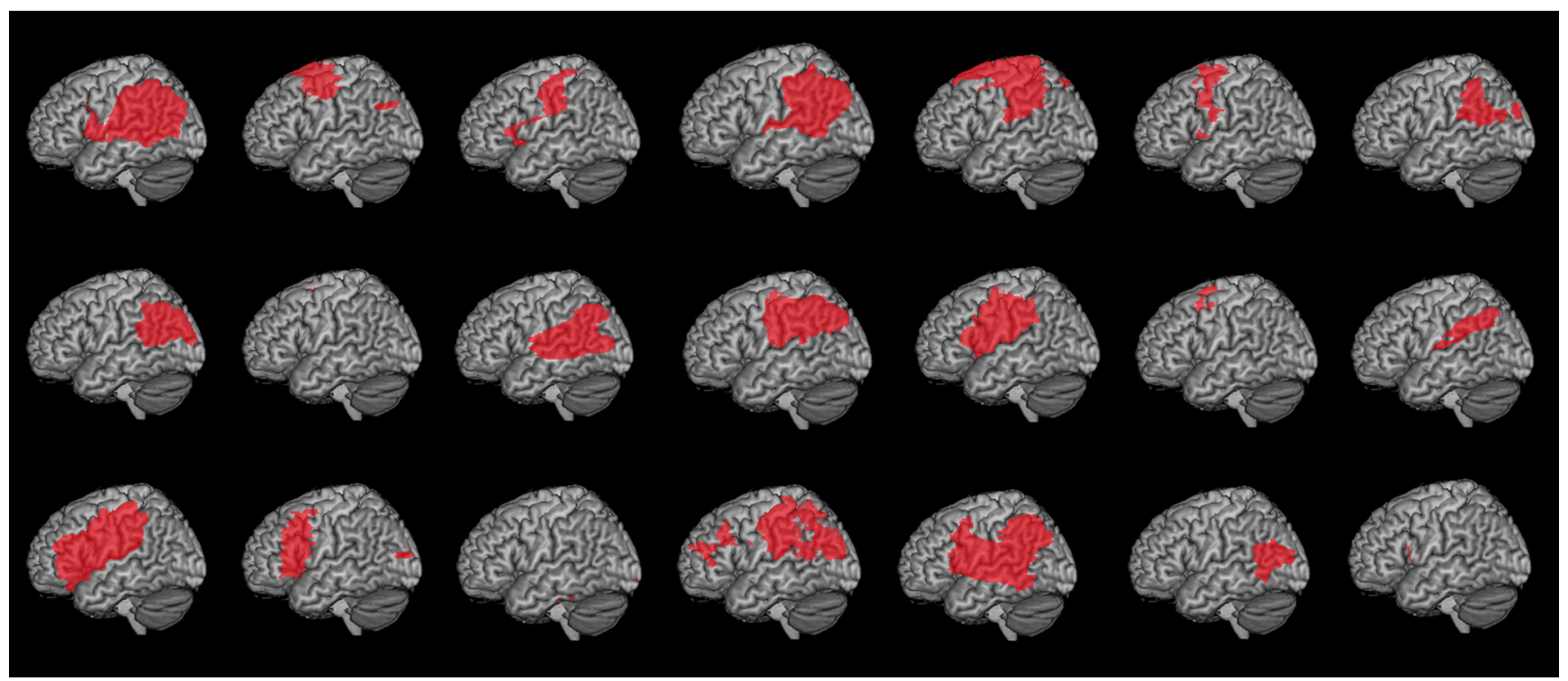

Figure 1. Cortical view of lesion maps for individual patients. Patients were selected to have a range of lesions spanning the left fronto-temporo-parietal cortex.

move before the end of the video, they were reminded to wait for the tone. The position of each participant's left index finger was continuously recorded using an Ascension TrakSTAR motion tracking system (Ascension Technology) at $150 \mathrm{~Hz}$.

Participants produced 16 trajectories (Fig. 2) grouped into two presentation conditions. We manipulated task difficulty by providing a differing number of visible landmarks $(2-\mathrm{cm}$ diameter circular dots placed on the Plexiglas) under the hypothesis that landmarks could potentially be used as reference points to subdivide a complex action into simpler point-topoint reaches, thus reducing the trajectory planning requirements. Stimuli were thus cued with reference to either a single central landmark or with four additional landmarks bounding a rectangular workspace. These landmarks do not necessarily occur at via points within every movement, but instead could be used as general points of reference for all trajectories. However, in general, we found that varying the number of landmarks did not significantly impact performance; thus, while we include a landmark factor in our statistical models for completeness (see Experimental design and statistical analysis), for conciseness, we do not report the outcomes of statistical tests of this factor in the Results.

More importantly, each trajectory was reproduced under two levels of stimulus type (body or cursor). As described in the Introduction, this stimulus-type manipulation was included to test whether trajectory imitation is dependent upon the ability to observe and represent the relative positions of limb segments during the action or whether trajectory imitation instead can be performed more abstractly by replicating only the kinematics of the endeffector. During the experiment, participants also saw a third "stick" stimulus-type (in which the actor's shoulder, elbow, and fingertip locations were used to render a segmented line representing the arm) to test how individuals imitate multijointed nonwell-defined landmarks.
1 landmark trajectories

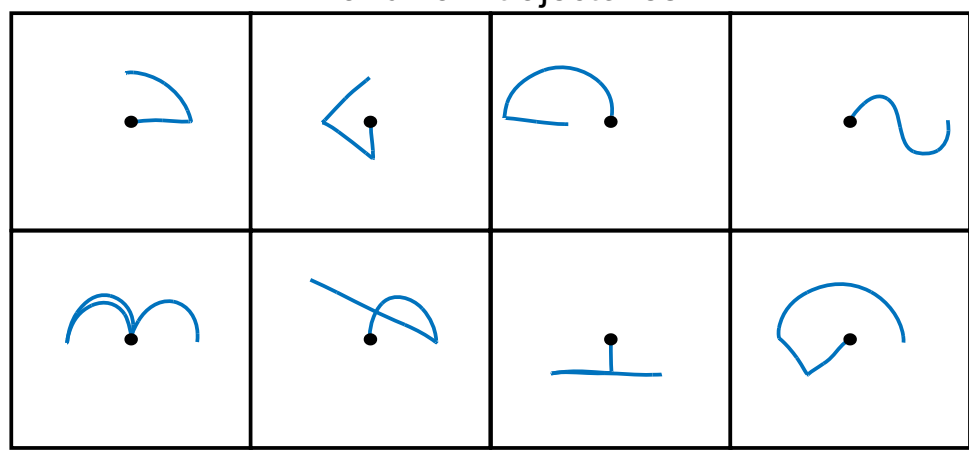

5 landmark trajectories

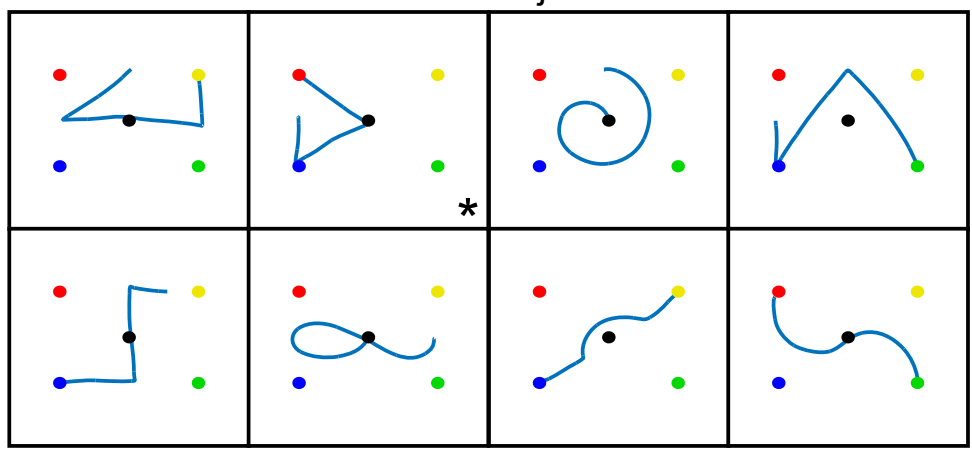

Figure 2. Reach trajectories used in the Production and Perception tasks. Trajectories were cued by observing the movement of either an actor or a cursor, and were made with reference to either one (top) or five (bottom) visible landmarks. The resulting static shapes derived from motion tracking of the actor's fingertip are shown above. In the one-landmark condition (top), motion always began at the central target; in the five-landmark condition (bottom), the starting location of the trajectory varied for each stimulus shape. In all cases, while participants observed a stimulus (actor or cursor) moving along these paths, a static representation of the trajectory path was never displayed as part of the stimulus. Second panel, the * denotes the trajectory from which the initial movement segment was extracted to evaluate the quality of imitating point-to-point reaches. This trajectory was selected because it contained a single straight line at the start of the movement that started and ended on

body stimuli. However, all participants found this condition confusing due to the projection of $3 \mathrm{D}$ arm motion into a $2 \mathrm{D}$ plane, as confirmed by reliably worse performance compared with the other conditions across all participants and tasks. Because this condition did not offer meaning- 
ful insight into trajectory imitation per se, we excluded it from analysis here.

Video stimuli for the body condition consisted of a seated actor producing trajectories on a Plexiglas board identical to the one in front of the participant (i.e., with the same number of landmarks). The actor was shown facing the participant and moving his right arm so that the experimenter's arm mirrored the participant's left arm. Participants were trained to imitate (in mirror) the actor; no mirror-inversion mistakes were observed for either the patients or the controls during this task. Video stimuli for the cursor condition consisted of a single black cursor moving over a white background, with landmarks visible as appropriate. Cursor trajectories were created from motion-tracking data collected during filming of the body stimuli, resulting in identical end-effector movement trajectories for the two stimulus types. Participants completed two blocks of trials: the two levels of the landmark factor ( 1 or 5 ) were manipulated between-blocks, whereas the two levels of the stimulus type factor (body or cursor) were intermixed within blocks.

As part of this Production task, we also examined the ability of patients and controls to imitate simple point-to-point reaches by evaluating the ability to imitate the initial segment of one of the more complex movement trajectories (see below for details). Analysis of point-to-point movements allows us to control for whether observed imitation deficits could be attributed to a lower-level deficit in movement execution.

Trajectory Perception task. A deficit in imitating trajectories could be attributed to one of two possible causes: (1) impairment in the ability to plan the necessary movement trajectory; or (2) difficulty with perceiving and/or recalling the required shape (i.e., a perceptual and/or working memory problem). To distinguish between these possibilities, participants were additionally required to complete a Perception task. In this task, participants were exposed to the identical video stimuli as in the Production task, but were asked to simply make a decision about the path they had observed rather than producing the actual movement. That is, stimuli consisted of two presentations of the same videos used in the Production task, followed by a $1 \mathrm{~s}$ delay before the presentation of a static image of a trajectory shape. Participants were instructed to indicate "if the path in the picture is the same as the movement made in the video." The shape either matched the exact trajectory produced in the video or was a mirror image of the trajectory that was reflected about the vertical or horizontal axis. The shape remained on the screen until participants indicated a match or nonmatch by pressing one of two buttons on a computer keyboard with their left hand. Task accuracy and reaction time (RT) were measured, although due to technical difficulties RT data at the trial level were unavailable for 1 control and 2 stroke participants; thus, these individuals could not be included in RT analyses involving mixedeffects models (see Experimental design and statistical analysis).

As with the Production task, participants in the Perception task completed two blocks of trials: the two levels of the landmark factor (1 or 5) were manipulated between-blocks, and the two levels of the stimulus type factor (body or cursor) were intermixed within blocks. The order of the Production and Perception tasks was counterbalanced across participants.

Participants were also required to complete a task intended as an independent test of visual working-memory ability with stimuli that were not trajectories. In this Memory control task, participants were shown one of eight images chosen from a set of abstract line drawings (Petrides and Milner, 1982). Each image was presented twice for $4 \mathrm{~s}$ with a $1 \mathrm{~s}$ delay in between; following a $1 \mathrm{~s}$ pause, a second stimulus was displayed, which was either identical or a rotated version of the first image, and participants were required to report whether the two images were the same or different using a keypress with their left hand. Thus, the structure of this task exactly mirrored that of the Perception task, except that the initial stimuli were static pictures instead of videos. Task accuracy and RT were measured using the same method as described below for the Perception task. Although average task accuracy did not differ between patients and controls ( $t$ test, $t_{(32)}=0.51, p=0.61$ ), average RT was different between groups ( $t$ test, $t_{(32)}=-2.67, p=0.01$ ) and so was introduced as a covariate when appropriate in the analyses involving RT below as well as in the Support-Vector Regression Lesion-Symptom Mapping (SVRLSM) analysis. The Production task data as well as the ROI LSM analyses were also reexamined with inclusion of average RT from the Memory task analysis as a covariate to control for any potential visual working memory deficits in the patient group, but as inclusion of this additional term did not change the significance of any findings, we do not report the results of those models here.

Stroke group auditory comprehension. Patients with strokes completed the auditory comprehension subsection of the Western Aphasia Battery (Kertesz, 1982), which quantifies capacity to understand verbal instructions.

Scores on the Western Aphasia Battery were high $(9.06 \pm 1.32$ of 10 possible points). Thus, we felt confident that any group differences in performance could not be explained by differences in instruction comprehension.

\section{Experimental design and statistical analysis}

All participants, as described above and in Table 1, completed both the Production task and the Perception task.

Production task. Response trajectories were recorded and analyzed offline using custom software written in MATLAB (The MathWorks). Fingertip velocity was calculated by smoothing the recorded fingertip position using a second-order Savitzky-Golay filter with a frame size of 19 samples, then taking the derivative. Movement start and end were identified as the time when the fore/aft reach velocity became minimal $(<0.03 \mathrm{~m} / \mathrm{s})$, reflecting the time when the participants contacted the Plexiglas surface and their primary axis of motion was along this surface. Each start and end time was verified by visual inspection to remove erroneous points, such as if a participant lifted his hand away from the Plexiglas in the middle of the trajectory. All motion produced between the identified start and end times was labeled as the complete imitated movement generated by the participant. The $3 \mathrm{D}$ data returned by the motion tracking system were projected into the plane of the Plexiglas surface to account for any small angular mismatch between the Plexiglas (on which the reference landmarks were positioned) and the vertical plane of the tracker axes. Movements were smoothed and timenormalized using a b-spline fit with 200 points. Finally, the shape of the generated response was compared with the ideal template (the normalized and smoothed trajectory produced by the actor in the stimulus video) by performing a Procrustes distance analysis (Goodall, 1991). The Procrustes distance analysis estimates the best translation, rotation, and scaling parameters that match the sample reach to the template, and returns a dissimilarity measure for the resulting transformed response trajectory shape (normalized between 0 and 1 , with 0 being identical and 1 being completely dissimilar). We opted to use this measure to provide an estimate of the ability for participants to reproduce all of the major features of the trajectory shape. While translation, rotation, and scaling could also be considered errors in imitation, it is difficult to completely dissociate true performance errors in these metrics from any small changes that arose from repositioning the tracker origin between participants; hence, we opted for a robust metric that quantifies errors after computing the best possible alignment of the performed action to the model template.

Data were analyzed using mixed-effects models, including fixed effects of Group (patient or control), Stimulus type (body-cued or cursorcued), Landmark (1-dot or 5-dot), Task order (Production first or Perception first), and random effects of Subject and Stimulus shape. Models were fit using the glmmTMB package in R (Magnusson et al., 2017) to improve convergence and to allow for the use of a logarithmic link function in the model to address the non-Gaussian (bounded between 0 and 1) nature of the Procrustes distance metric. Significant main effects were evaluated using likelihood ratio tests comparing a model with the effect of interest against a model without the effect of interest (which approximately follows a $\chi^{2}$ distribution). Step-down Bonferroni-Holm corrections were applied to address multiple comparisons across likelihood ratio tests. Here and throughout, error bars indicate SEM.

Trajectory Production control:point-to-point reaches. Although patients were tested on their nonparetic side, we wanted to confirm that errors made during trajectory Production could not be explained by a low-level motor execution deficit. Thus, we isolated the point-to-point reach comprising the initial movement segment of one of the five landmark stimuli 
for both the body and cursor conditions (Fig. 2, triangular shape denoted with an asterisk). This particular stimulus was chosen because it afforded a reasonably long initial movement segment that started and ended on visible landmarks, thus providing clearly identified movement start and end positions that defined the desired movement. The movement segment of interest was identified using a velocity threshold (tangential velocity $>0.05 \mathrm{~m} / \mathrm{s}$ ) and was verified by visual inspection to address cases where participants did not completely pause between segments. To evaluate the quality of reproducing the point-to-point movement, we calculated the maximal reach deviation as the greatest absolute perpendicular deviation of the finger from the straight line between the initial and final fingertip position of the point-to-point reach. We also calculated the initial reach direction of the velocity vector $100 \mathrm{~ms}$ after movement onset, and the initial direction error as the signed difference between the initial reach direction of the participant and the ideal (actor) reach direction.

Data were analyzed with mixed-effects models with main effects of Group and Stimulus type (body-cued or cursor-cued), and random effect of Subject regressing against maximal reach deviation from a straight line or initial direction error. Models were fit using the lme 4 package in $\mathrm{R}$ (Bates et al., 2015). Significant effects were evaluated using likelihood ratio tests.

Perception task. RT was measured as the time between the onset of the static trajectory and initiation of a button-press response (reporting "same" or "different"). RT data were trimmed by first removing the RTs on incorrect trials (because incorrect responses might arise from nontask-related causes, e.g., lack of attention on a particular trial), then removing all RT outliers falling $>2.5$ SDs of the mean. Accuracy and RT were analyzed with mixed-effects models with main effects of Group, Stimulus type (body-cued or cursor-cued), Landmark (1-dot or 5-dot), and Task order (Production first or Perception first), and random effects of Subject and Stimulus shape. In the case of RT, average RT performance in the Memory task was also included as a fixed effect to control for individual differences associated with working memory. For all behavioral tests, the logarithm of the RT $(\operatorname{logRT})$ was used to reduce outliers and address the long-tail distribution, as it is well recognized that the $\log$ RT distribution is approximately Gaussian (e.g., Carpenter, 1981). Models were fit using the glmmTMB package in R (Magnusson et al., 2017), using a Gaussian link function in the case of RT or a binomial family in the case of Accuracy. Significant main effects were evaluated using likelihood ratio tests comparing a model with the effect of interest against a model without the effect of interest; step-down BonferroniHolm corrections were applied to address multiple comparisons.

Correlations in performance between the Perception and Production tasks were tested by examining the relationship between mean Procrustes distance and mean accuracy or mean $\operatorname{logRT}$, with means taken within each subcondition (i.e., for each unique Stimulus type and Landmark). Means were used in this analysis to allow for calculation of a Percentage Accuracy score in the Perception task while preserving potential differences arising from the effects of stimulus type or landmark. Mixed-effects models also included fixed effects of Group (patient, control), Stimulus type (body-cued or cursor-cued), and Landmark (1-dot or 5-dot), and random effects of Subject. Models were fit using the lme4 package in $\mathrm{R}$ (Magnusson et al., 2017). Significant main effects of the effect of interest (i.e., Accuracy or RT) were evaluated using likelihood ratio tests comparing a model with the effect of interest against a model without the effect of interest.

Lesion location identification. T1-weighted MRI brain scans (15 patients) or CT scans without contrast (if MRI was contraindicated, 6 patients) were used to identify lesion locations; data from all 21 patients were included in the analyses listed below. Lesions were segmented under the supervision of a neurologist ( $\mathrm{H}$. Branch Coslett) who was blind to all behavioral data. Details of the lesion location identification procedure can be found in Kalénine et al. (2010). Individual lesion locations for all stroke participants are shown in Figure 1. Total lesion volume for each individual patient was calculated $\left(71,850 \pm 34,488 \mathrm{~mm}^{3}\right.$; Table 1$)$ and used in some analyses as a control for overall stroke severity (see below).

Multivariate LSM. Multivariate LSM was conducted using SVR-LSM (Zhang et al., 2014), a machine learning technique for multiple regres- sion that trains a model to predict behavioral scores using the full lesion maps of all participants. SVR-LSM is an improvement over earlier techniques, such as voxel-based LSM (Bates et al., 2003), which perform all analyses at the individual voxel level and then aggregate across voxels to create a full-brain map. By predicting behavioral scores using all voxels simultaneously, SVR-LSM has the capability to avoid vasculature-related biases that can systematically distort lesion-symptom maps (Mah et al., 2014). SVR-LSM analyses were performed using software written by Zhang et al. (2014) (https://cfn.upenn.edu/ zewang/) and modified by DeMarco and Turkeltaub (2018). As this is a relatively new technique, we validated our findings using more traditional univariate voxel-based LSM approaches (Kalénine et al., 2010, 2013; Watson and Buxbaum, 2015) and obtained qualitatively similar results (results not reported). As with the behavioral analyses, mean RT during the Memory Task was included as a behavioral covariate in the SVR-LSM analysis to control for any differences in working memory across patients.

Statistical significance was determined using a voxelwise threshold. First, a $\beta$ map of the observed data was computed. This $\beta$ map regressed out the effects of total lesion volume in both the behavioral and lesion data to avoid identifying spurious voxels whose lesion status correlates with lesion volume (DeMarco and Turkeltaub, 2018); this approach has been reported to address mislocalization of statistically significant regions due to vascular-based biases. Only voxels damaged in at least 3 participants (15\% of the stroke group) were included in the analyses. Although this voxel-inclusion criterion is frequently set to $10 \%$ of the total number of patients (Kalénine et al., 2010), due to our small sample size, we increased this threshold to $15 \%$ to decrease the number of spurious voxels included in the analysis. A voxelwise $p$ map was then derived by comparing the observed $\beta$ map to $\beta$ maps from 10,000 Monte-Carlo style permutations where behavioral scores were randomly paired with lesion maps. By using 10,000 permutations, we have greater confidence that we have adequately approximated the null $\beta$ value distribution for purposes of permutation testing, providing greater confidence in our $p$ value estimates. Finally, we applied a multiple-comparisons correction to our data. Because SVR-LSM is a multivariate technique in which $\beta$ values are calculated across all voxels simultaneously, but is univariate in the sense that $p$ values are estimated for each voxel independently, it remains unclear exactly how best to correct for multiple comparisons (Mirman et al., 2018; Sperber et al., 2018). Thus, for analyses averaging across the body and cursor conditions, we applied a cluster-level false-discovery rate (FDR) correction to our maps of $p$ values. Specifically, we searched for clusters ( $>250$ voxels) of similar $p$ values (nonoverlapping bins of $p$ values in increments of 0.0005$)$ using the spm_clusters function in SPM12 (Penny et al., 2007), and submitted the $p$ values associated with those clusters to an FDR correction $(q=0.1)$. The outcomes of this analysis were consistent with the results of a univariate voxel-based LSM approach followed by an FDR correction (data not shown). As an exploratory analysis, we were also interested in visualizing the potential overlap in brain regions correlated with behavior within the body or cursor conditions separately; results for these analyses were shown at an uncorrected threshold of $p<0.05$ followed by a minimum-cluster-size correction (removing clusters $<500 \mathrm{~mm}^{3}$ ).

In addition, we performed ROI analyses to confirm whether there was any relationship between performance in either the Production or Perception task and the percentage of lesioned voxels within three ROIs selected a priori: Brodmann area 6 (premotor cortex), Brodmann areas 39 (angular gyrus), and Brodmann area 40 (supramarginal gyrus). This complementary approach allowed us to investigate whether behavior was correlated to consistently damaged brain regions rather than to individual voxels, which may not always be damaged within the same region across individuals. Thus, agreement between these models and our SVRLSM results gave us increased confidence that we were not simply reporting false positives. Mixed-effects models were run using the $\operatorname{glmm} T M B$ package in R (Magnusson et al., 2017) and, aside from the three preselected ROIs, also included fixed effects of Total Lesion Volume, Stimulus type (body or cursor), and Landmark (1-dot or 5-dot). Models also included random effects of Subject and Stimulus shape. Significant main effects were evaluated using likelihood ratio tests comparing a model with the effect of interest against a model without the effect of interest, 

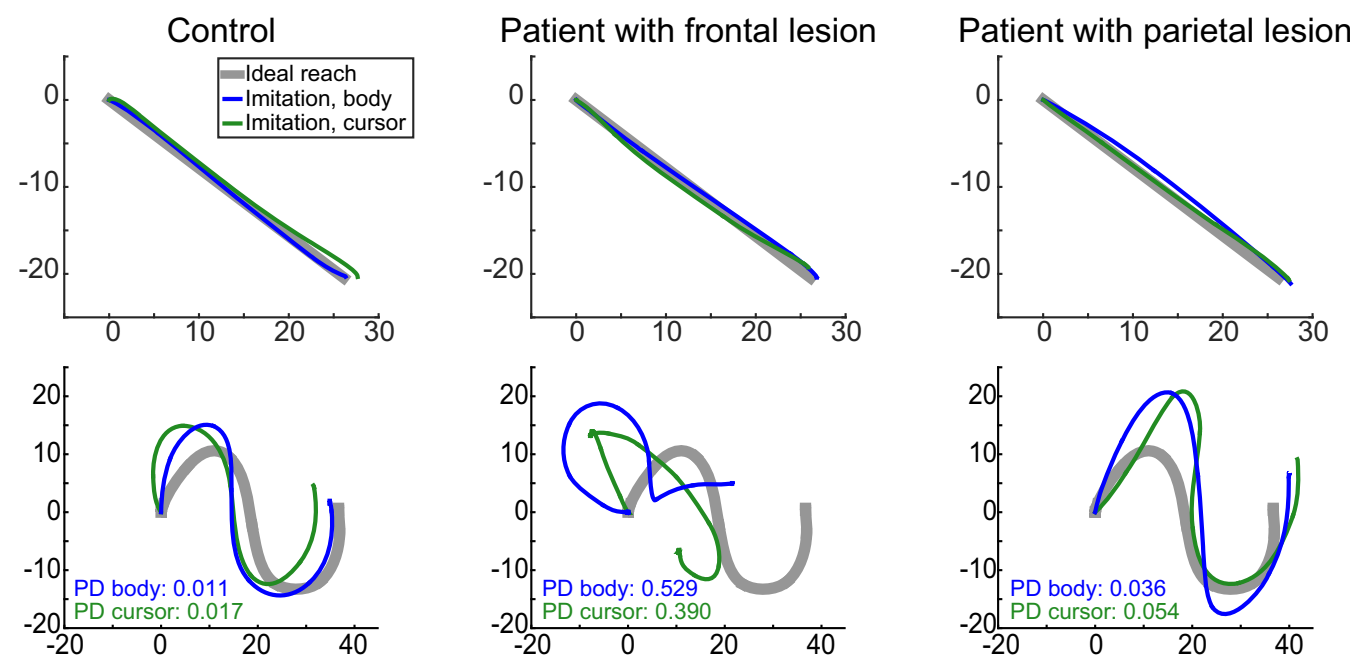

Figure 3. Examples of imitated trajectories from a single control participant (left column), a patient with dorsal premotor but without inferior partial damage (center column), and a patient with inferior parietal but not dorsal premotor damage (right column). Trajectories were chosen to clearly illustrate what good or bad performance looked like across patients compared with controls. All panels, Blue represents imitation of a body stimulus. Green represents imitation of a cursor stimulus. Thick gray line indicates the ideal movement as performed by the actor. Point-to-point trajectories were comparable across participants (top row). In contrast, imitation ability for complex paths (bottom row) was worse for the patient with dorsal premotor damage than the other 2 participants, for both the body and cursor conditions. Imitation ability was quantified using a shape dissimilarity (Procrustes distance [PD]) metric, in which larger values indicate worse replication of the actor's movement.

with step-down Bonferroni-Holm corrections applied to address multiple comparisons.

\section{Results}

Twenty-one patients with left-hemisphere stroke (Fig. 1) and 13 age-matched controls were asked to imitate meaningless trajectories (Fig. 2) using their left (nonparetic, nondominant) hand after watching the movement of either an actor (body-cued stimulus) or a cursor (body-free stimulus). Performance in this Production task was contrasted against a Perception task in which they simply had to report whether a subsequently displayed static image had the same or different shape compared with the stimulus trajectory.

\section{Patients and controls exhibited comparable point-to-point reach kinematics}

We first analyzed the imitation of point-to-point reaches to confirm that the ability to reproduce straight movement paths was unimpaired in patients. This movement was the first segment of a more complex trajectory, which comprised a single straight movement that began and ended on physical landmarks in the workspace. Example point-to-point movements for a representative control, a patient with dorsal premotor but without inferior parietal lobe damage (Fig. 1, Patient 6), and a patient with inferior parietal lobe damage but without dorsal premotor damage (Fig. 1, Patient 1) are shown in Figure 3. As can be seen by comparing the three top panels of the figure, all 3 participants produced spatially similar movements. This observation was confirmed in a group-level analysis focusing on the ability to generate reaches that were straight and aimed in the correct direction. Specifically, we measured the straightness of the movement path (maximum hand-path deviation from a straight line) and the initial reach direction error (deviation of the aiming direction of the hand $100 \mathrm{~ms}$ after movement initiation). The patient and control groups did not differ in either the straightness of the movement path (patients: $4.28 \pm 2.91 \mathrm{~mm}$; controls: $3.49 \pm$ $\left.1.72 \mathrm{~mm} ; \chi_{(2)}^{2}=3.26, p=0.20\right)$ or the initial direction error (patients: $3.48 \pm 2.96^{\circ}$; controls: $5.39 \pm 1.79^{\circ} ; \chi_{(2)}^{2}=0.25, p=$
0.62). This is not surprising, as patients with apraxia tend to have relatively unimpaired reaching and grasping ability in their nonparetic hand (Buxbaum et al., 2005; Ietswaart et al., 2006) unlike the difficulty in generating straight movements with the paretic limb typically observed in stroke (Dewald et al., 2001; Cirstea et al., 2003; Osu et al., 2011). Thus, patients and controls imitated point-to-point reaches with comparable accuracy, suggesting that any imitation abnormality with respect to more complex (curved and/or multisegmented) trajectories cannot be attributed to an abnormality in movement execution.

\section{Imitation of complex trajectories was impaired in patients with left hemisphere damage}

Despite being able to produce straight point-to-point trajectories, patients with left hemisphere damage exhibited deficits in the ability to reproduce the shapes of movement trajectories in the Production task (see Materials and Methods). Example trajectories are shown in Figure 3 (bottom panels). Shape imitation errors were quantified by calculating a dissimilarity score (Procrustes distance metric) (Goodall, 1991) between the movement of the actor/cursor displayed in the stimulus and the trajectory produced by the participant. Mean dissimilarity scores are shown in Figure 4 at both the group (bars) and participant (lines) level. Qualitatively, although patients exhibited a wide range of dissimilarity scores, as a group, their shape dissimilarity scores were generally larger than those of the controls but similar between the body-cued or cursor-cued stimuli.

When shape dissimilarity scores were analyzed with a mixedeffects model, there was a main effect of group (patient vs control: $\left.\chi_{(2)}^{2}=17.29, p=0.0009\right)$, in which stroke participants reproduced the shape less accurately compared with controls. However, no significant main effect was observed for stimulus type (body-cued vs cursor-cued: $\chi_{(1)}^{2}=0.613, p=0.434$ ), suggesting that, regardless of whether or not the stimuli provided information about the relative positioning of limb segments during movement, participants performed equally well at imitating the required shape. 


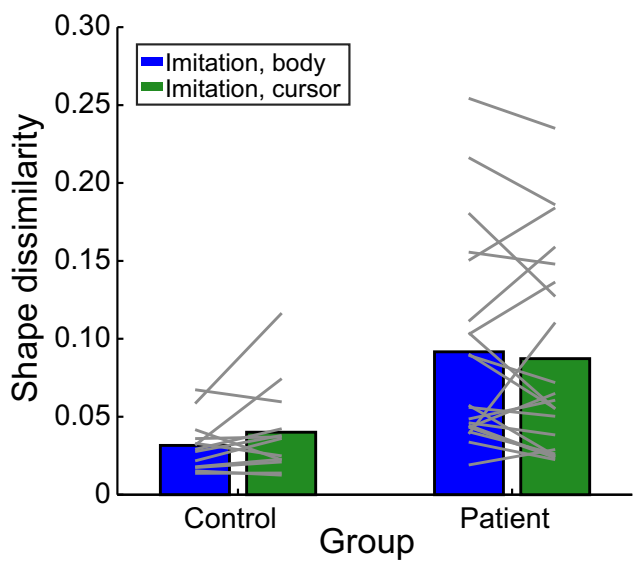

Figure 4. Mean shape dissimilarity (Procrustes distance) of imitated movements in the Production task. Group means (bars) and individual means (lines) are shown separately for the body-cued (blue) and cursor-cued (green) conditions, for both patients and controls.

Consistent with the lack of a main effect of stimulus type on imitation performance, at the individual level, participants who exhibited larger shape dissimilarity scores in the body condition also performed worse during the cursor condition. Regression analyses indicated that participants' average shape dissimilarity in the body task was highly correlated with their average shape dissimilarity in the cursor condition, with a slope near 1 and intercept near $0\left(R^{2}=0.78\right.$; slope $=0.902$, intercept $\left.=0.006\right)$. Similar results were obtained when considering only the stroke participants $\left(R^{2}=0.78\right.$; slope $=0.88$, intercept $\left.=0.015\right)$. Thus, the ability to imitate trajectory paths was not different whether participants were given information about the configuration of the entire limb or saw only a body-independent representation of the end-effector.

We also found that average trajectory-imitation performance for both the body and the cursor conditions in patients correlated with performance on a more conventional meaningless imitation task (Watson and Buxbaum, 2015) that cues imitation based on the motion of an actor (Table 1; body condition: $R^{2}=0.33, p=0.006$; cursor condition: $\left.R^{2}=0.25, p=0.02\right)$, suggesting that, in general, performance was consistent across different meaningless-imitation tasks.

Although patients also moved more slowly than controls when imitating trajectories (peak velocity: controls, $0.40 \pm 0.056$ $\mathrm{m} / \mathrm{s}$; patients, $\left.0.37 \pm 0.056 \mathrm{~m} / \mathrm{s} ; \chi_{(2)}^{2}=9.25, p=0.009\right)$, a group difference in shape dissimilarity remained even after accounting for this effect $\left(\chi_{(2)}^{2}=7.83, p=0.005\right)$, and there were no significant differences in either acceleration or jerk between the two groups $(p>0.26)$. Thus, it was not possible to explain these group-level differences on the basis of simpler kinematic parameters, consistent with the lack of an observed impairment at imitating straight movements as noted above. Finally, patient performance could not be explained by a nonspecific cognitive effect of lesion volume, as there was no relationship between shape dissimilarity and total lesion volume $\left(R^{2}=0.001, p=\right.$ 0.857).

Trajectory Perception was also impaired in patients with lefthemisphere damage

One potential concern was that imitation deficits could arise not because of problems in generating the desired action, but as a result of deficits in perceiving or remembering the movement to be copied. The findings reported here are unlikely to reflect a more general visual working memory deficit or difficulty in mental manipulation of stimuli based on an additional control task (see Materials and Methods). Hence, here we were primarily concerned with whether there was a problem in perceiving the visual motion of the stimulus and transforming it into the shape of the trajectory path. To examine this, participants were required to complete a Perception task in which they observed a movement trajectory as in the Production task, but then simply had to make a binary choice (yes/no) as to whether a subsequently displayed static image had the same shape as the path of the viewed stimulus trajectory. Foils were static images of the correct path shape that were then reflected about the horizontal or vertical axis; as there was no significant effect of reflection direction on performance (accuracy: $\chi_{(1)}^{2}=0.738, p=0.39$; RT: $\chi_{(1)}^{2}=1.00, p=0.32$ ), the data presented below were collapsed across reflection directions. As before, the stimuli could be cued either by an actor or a cursor.

Mean accuracy and RT in identifying a static shape as being the same or different from the observed movement are shown in the Figure $5 A$ and Figure $5 B$, respectively. As with the Production task, patients exhibited a wide range of performance but were generally worse than controls (lower accuracy, higher RT) despite a potential ceiling effect on performance accuracy. Accuracy and RT measures were negatively correlated across participants $\left(r^{2}=\right.$ $0.25, p=0.004)$, suggesting that impairments in trajectory Perception generally affected both measures similarly. However, because accuracy is informative about the likelihood of correctly reporting the observed trajectory, whereas RT as a continuous measure provides a sense of uncertainty about that choice, both provide complementary information about performance in this Perception task.

For response accuracy (Fig. 5A), a mixed-effects model revealed no significant main effects: while there was a small difference between groups $\left(\chi_{(1)}^{2}=5.229\right)$ and between stimulus types (body-cued vs cursor-cued: $\chi_{(1)}^{2}=3.951$ ), neither effect was significant after correcting for multiple comparisons $(p=0.11$ and $p=0.19$, respectively). No significant interaction was observed between group and stimulus type $\left(\chi_{(1)}^{2}=0.445, p=0.45\right)$.

For RT (Fig. 5B), a mixed-effects model showed a significant main effect of group $\left(\chi_{(2)}^{2}=14.89, p=0.0023\right)$, with patients having longer RTs than controls $(2623.7 \pm 948.3 \mathrm{~ms}$ and $1638.4 \pm 696.0 \mathrm{~ms}$, respectively). We observed no effect of testing order $\left(\chi_{(1)}^{2}=2.49, p=0.114\right)$ or any interaction between order and group or stimulus type. Importantly, we again observed no significant effect of stimulus type $\left(\chi_{(1)}^{2}=2.633, p=0.209\right)$ and no interaction between stimulus type and group $\left(\chi_{(1)}^{2}=0.450\right.$, $p=0.502)$. Together, the accuracy and RT data suggest that patients, in general, exhibited disrupted performance on the Perception task compared with that of control participants but performed similarly whether the movement was cued by an actor or a cursor. Thus, as with the Production task, availability of additional information about the relative positioning of the limb segments did not strongly influence the ability to accurately perceive and report the observed trajectory path.

\section{Performance on the Production and Perception tasks was uncorrelated}

The previous two analyses indicated that left hemisphere damage disrupts performance on both the Production and Perception tasks. Because these analyses were performed at the group level, we wanted to examine whether the same participants exhibited deficits on both tasks (indicating that both tasks potentially rely on the same underlying computational processes, or that difficulties in the Perception task can account for impaired performance 
A

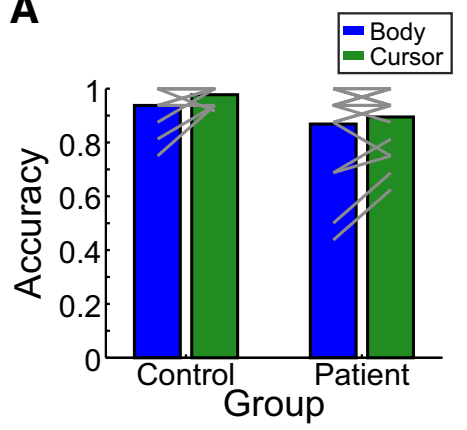

B

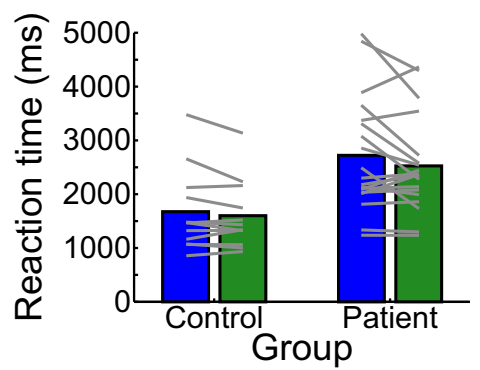

Figure 5. Mean performance in the Perception task was quantified using the complimentary measures of accuracy $(\boldsymbol{A})$ and RT $(\boldsymbol{B})$. Group means (bars) and individual means (lines) are shown separately for the body-cued (blue) and cursor-cued (green) conditions, for both patients and controls.

A

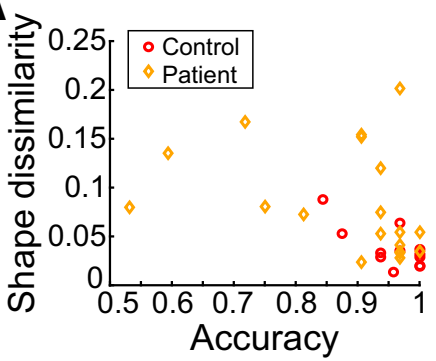

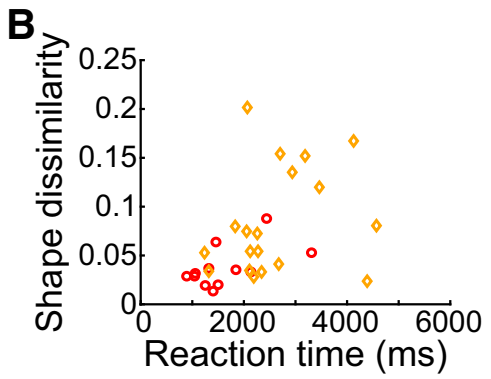

Figure 6. Relationship between Production (ordinate) and Perception (abscissa) tasks, shown separately for Perception task accuracy $(\boldsymbol{A})$ and RT $(\boldsymbol{B})$. Mean performance is shown for each patient (orange diamonds) and control (red circles).

0.12). Thus, across both groups, we observed a dissociation between how participants performed on the Perception and Production tasks, which suggests that at least some participants had difficulties specifically in the ability to imitate but not to perceive the shape of the desired movement.

\section{Lesion analyses}

Given the heterogeneity of patient performance in both the Production and Perception tasks, our final set of analyses focused on understanding this variability based on lesion location. We used two complementary lesion-analysis approaches. First, we performed SVR-LSM analyses (Zhang et al., 2014) to examine the voxel-level relationship between performance in the behavioral tasks (shape dissimilarity for the Production task and both the accuracy and RT measures for the Perception task) and the patients' lesion locations. Second, we performed an ROI analysis (Buxbaum et al., 2014) to examine the contributions of specific brain areas (defined on the lesion maps according to Brodmann areas) that we hypothesized to be involved in imitation based on prior research; this included PMd (approximated using Brodmann area 6 , al-

in the Production task), or whether the group-level deficits stemmed from different subgroups of patients that were impaired on only one of the two tasks (suggesting that separate processes may underlie performance of the Production and Perception tasks).

The relationships between shape dissimilarity in the Production task and performance (accuracy and RT) in the Perception task are summarized in Figure 6. This figure suggests that there is no clear relationship between performance on the two tasks. Indeed, several patients exhibited relatively good performance in the Production task (low shape dissimilarity) but disrupted performance on the Perception task (low accuracy/high RT), whereas other patients exhibited the opposite pattern. Indeed, of those patients who performed poorly on the Production task, their performance in the imitation task ranged from quite poor to as good as controls.

To confirm these impressions and to guard against outliers more robustly, we used mixed-effects models to predict mean Production-task shape dissimilarity using either mean Perception-task accuracy or mean Perception-task RT (see Materials and Methods). These models also accounted for the effects of primary task factors (e.g., group and stimulus type) and a random effect of participant and, in the case of RT, also accounted for performance on the Memory control task. In this analysis, we found no significant effect of Perception task accu$\operatorname{racy}\left(\chi_{(1)}^{2}=1.07, p=0.30\right)$ or $\mathrm{RT}\left(\chi_{(1)}^{2}=0.070, p=0.79\right)$ on shape dissimilarity, suggesting that how well a given individual performed on the Production task was uncorrelated with their performance during the Perception task. This was also true when looking only at patients (effect of accuracy: $\chi_{(1)}^{2}=0.028, p=$ 0.37; effect of RT: $\chi_{(2)}^{2}=0.24, p=0.62$ ) or only at controls (effect of accuracy: $\chi_{(2)}^{2}=0.027, p=0.87$; effect of RT: $\chi_{(2)}^{2}=2.37, p=$ though we recognize that Brodmann area 6 also includes ventral premotor cortex) (Hocherman and Wise, 1990; Caspers et al., 2010; Pearce and Moran, 2012), and IPL (Brodmann areas 39 and 40) (Buxbaum et al., 2014; Kadmon Harpaz et al., 2014). The ROI analyses allowed us to identify correlations between damage to larger neural regions and behavioral impairments: effects that might be missed with a voxel-level analysis, such as SVR-LSM, particularly if there is intersubject variability in the individual voxels associated with the task. Importantly, the results of both lesion analysis approaches were found to be consistent; we report the outcomes of both approaches below.

The SVR-LSM analysis of lesions associated with shape dissimilarity in the Production task (averaging across stimulus type) is shown in Figure 7A. The lesions contributing most highly to increased shape dissimilarity were located in the dorsal premotor and primary motor cortices. These regions were highly overlapping for the body and cursor conditions when analyzed separately (Fig. $7 B$ ), although there was some suggestion that regions associated with poor performance in the body-cued condition extended more ventrally whereas those in the cursor-cued condition extended more dorsally.

In contrast, the SVR-LSM analyses for the Perception task suggested that accuracy in reporting movement shape is associated with damage to the supramarginal gyrus (Fig. 7C). Interestingly, although there was a high degree of overlap of lesions associated with worse accuracy in the body-cued and cursor-cued conditions separately (Fig. 7D), areas associated with worse performance in the body-cued condition were much larger and extended ventrally to encompass areas traditionally associated with the ventrodorsal stream and imitation ability (Buxbaum et al., 2014). We did not observe any significant regions that survived 


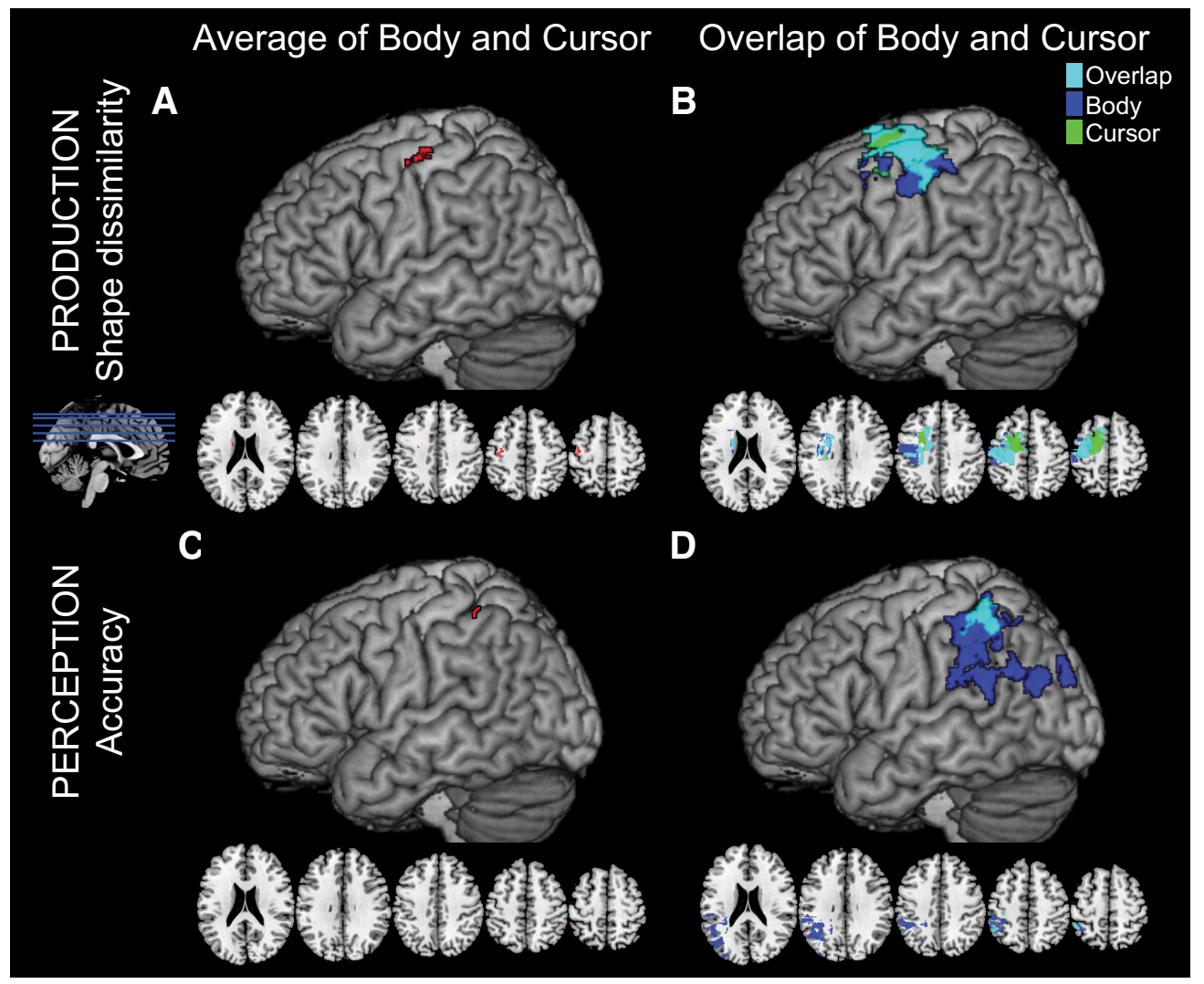

Figure 7. Results of the SVR-LSM analyses $(N=21)$ of shape dissimilarity in the Production task $(\boldsymbol{A}, \boldsymbol{B})$ and Perception task accuracy $(\boldsymbol{C}, \boldsymbol{D})$. Results are presented for both the average data collapsing across the body and cursor stimuli (left column; cluster-level FDR-corrected for multiple comparisons), and an exploratory analysis looking at regions associated with the body and cursor results separately as well as their overlap at a relaxed statistical threshold (right column; not corrected for multiple comparisons).

multiple-comparisons corrections for the SVR-LSM analysis of the Perception task RT data.

Thus, in general, the findings from the SVR-LSM analysis suggest that lesions to different regions of the brain were associated with performance in the Production and Perception tasks, respectively. The Production task was largely correlated with lesions in PMd and M1, whereas the Perception task was associated with lesions in the IPL. Furthermore, in both tasks, lesions that were associated with behavioral deficits due to cuing using a body-based or body-free stimulus substantially overlapped. However, there was a tendency for the regions associated with deficient performance with body stimuli to be more inferior than those associated with the overlap of body and cursor conditions, particularly in terms of accuracy performing the Perception task.

The SVR-LSM analysis looks for consistently lesioned continuous groups of voxels across all subjects that are associated with behavioral impairments. However, because of our relatively small sample size and the fact that stroke lesion sites do not follow predefined brain regions, we verified our findings using an ROI analysis approach using regions selected $a$ priori that included PMd (Brodmann area 6, PM) and the IPL (Brodmann areas 39 and 40). In doing so, we found results consistent with the SVR-LSM analysis. Specifically, for the Production task, the proportion of damage to Brodmann area 6 (premotor cortex) significantly predicted shape dissimilarity $\left(\chi_{(1)}^{2}=6.22, p=0.050\right)$. In contrast, for the Perception task, there was a significant correlation between accuracy and proportion damage to Brodmann area 40 (supramarginal gyrus) $\left(\chi_{(1)}^{2}=6.67, p=0.039\right)$. We did not observe any other correlations between these regions and the performance metrics from the Production or Perception tasks, and there was no significant effect of stimulus type in any cases.

Together, the results of the two lesion-analysis approaches provide evidence of correlations between lesions in PMd with impaired performance in the Production task, and correlations between lesions in IPL with impaired performance in the Perception task.

\section{Discussion}

Although imitation is an important aspect of learning, communicating, and using tools, the mechanisms by which people imitate are poorly understood. Here we demonstrated that meaningless actions can be imitated by representing the trajectory of the end-effector's movement through space, and that this ability is disrupted by a stroke affecting the left PMd: imitation of trajectories cued by an actor or a cursor become similarly impaired. Such impairments cannot be attributed to motor execution deficits due to stroke because they were observed in the nonparetic arm and patients had no difficulty imitating point-to-point gestures. These findings support the existence of a bodyindependent, trajectory-based route for imitation.

\section{Imitation by representing the trajectory path}

In our task, patients and controls performed similarly when imitating trajectories cued by an actor (providing information about whole-limb configurations) or a cursor (providing information only about end-effector motion). Thus, gestures can be imitated by representing only the trajectory path of the movement, consistent with research suggesting that participants 
attend only to the portion of the arm most relevant for imitation (Matarića and Pomplunb, 1998). Interestingly, a bodyindependent trajectory-based route for imitation could explain how species, such as dolphins or parrots, imitate humans (Taylor and Saayman, 1973; Moore, 1992; Bauer and Johnson, 1994) despite lacking homologous limbs.

Previous research has provided evidence that movements are planned as end-effector trajectories (Abend et al., 1982; Flash and Hogan, 1985). For example, hand position variance is smaller than that of the elbow or shoulder (Sergio and Scott, 1998; Tseng et al., 2002). Our own work suggests that trajectory paths are used to plan reaches around obstacles (Wong et al., 2016), and they have also been shown to guide corrective responses to unexpected perturbations (Cluff and Scott, 2015). Finally, trajectory representations can explain the kinematic invariance of letters written with different effectors (Wright, 1990; Rijntjes et al., 1999) or at different sizes (van Galen and Teulings, 1983; Wing, 2000).

Gestures could be represented compactly by specifying only end-effector movement trajectories, a more computationally tractable approach than recalling the motion of every limb joint (Ijspeert et al., 2001; Billard et al., 2004). Furthermore, because trajectory representations are not directly tied to the details of movement execution (e.g., controlling particular muscles), a single representation can support movement with either arm; this provides one potential explanation for how a unilateral lefthemisphere lesion could disrupt movement imitation bilaterally.

Finally, our data indicate that PMd is associated with the ability to plan trajectory paths. This region has previously been associated with the dorsodorsal stream (Rizzolatti and Matelli, 2003; Binkofski and Buxbaum, 2013), which is primarily associated with planning visually guided reaches. However, the dorsodorsal stream has also been associated with imitation (Rumiati et al., 2005; Caspers et al., 2010; Hoeren et al., 2014), and neural activity in PMd modulates with hand trajectory (Hocherman and Wise, 1990, 1991; Pearce and Moran, 2012; Pilacinski et al., 2018). Previous studies probing trajectory imitation have also implicated the importance of the left hemisphere (Platz and Mauritz, 1995; Weiss et al., 2001), although limitations in those studies have made it challenging to correlate performance deficits to specific brain regions. Our findings thus suggest that PMd, and the dorsodorsal stream in general, is important for planning actions when the goal can be specified in a body-independent manner (e.g., a location in space or trajectory path).

Planning trajectory paths could be more broadly useful for praxis (imitating gestures, pantomiming, or using tools). Tool use, for example, could be guided by planning the tool's trajectory rather than (or in addition to) the potentially incongruous movement of one's body. Such a trajectory representation is consistent with previous descriptions positing that imitation and tool-use abilities depend upon representations of "time-spaceform pictures" of movements (Liepmann, 1905; Geschwind, 1975; Mack et al., 1993).

\section{Dissociation between Production and Perception}

In addition to trajectory-imitation deficits, patients with stroke also had difficulties reporting the shapes of observed trajectories. Although we did not directly test whether these difficulties arose from an underlying spatial processing deficit, our memory control task as well as the work of others suggest that this is unlikely (Weiss et al., 2001). Interestingly, patients who performed poorly at producing trajectory paths exhibited a wide range of abilities in accurately reporting trajectory shapes. Moreover, these tasks appeared to dissociate neuroanatomically: deficits in the Produc- tion task were associated with lesions in PMd (part of Brodmann area 6), whereas behavioral deficits in the Perception task were associated with lesions in the IPL (supramarginal gyrus, Brodmann area 40), even after controlling for potential visual working memory impairments. Together, this hints that there may be at least a partial dissociation between the Perception and Production tasks.

While impaired performance in only the Production task could be easily explained as a disruption at a later planning stage, it is more challenging to explain why some participants appeared to be impaired only in the Perception task. We speculate that this dissociation could arise for a couple of reasons. First, the Perception task requires recognizing the similarity between the stimulus movement and a static shape, whereas the Production task requires recall of the movement. Therefore, the lack of correlation between the Production and Perception tasks would be consistent with the distinction between recognition and recall (Hollingworth, 1913; Kintsch, 1970; Anderson and Bower, 1972; Tulving, 1976). Second, the Perception task may uniquely require additional processing stages, such as transforming dynamic movements into static images (Korneev and Kurgansky, 2014), or distinguishing mirror-reversed shapes (Davidoff and Warrington, 2001), processes associated with the parietal cortex.

\section{Two routes to imitation}

Despite the benefits of imitating trajectories, under certain circumstances it is necessary to copy the configuration of the entire limb. For example, a coach may instruct an athlete to modify her elbow position while swinging a tennis racket. Using a trajectorybased approach would require separate representations for both the racket and the elbow (Ijspeert et al., 2001), and it has been shown that planning multiple trajectories simultaneously is challenging (Albert and Ivry, 2009). In such situations, it makes more sense to simply specify the transformations required to reconfigure the entire limb (Chaminade et al., 2005; Amorim et al., 2006): that is, to represent body configurations rather than trajectories (Goldenberg, 1999, 2013; Buxbaum et al., 2000; Schwoebel et al., 2004). Indeed, evidence for a body-dependent route to imitation comes from studies in which imitation ability is scored according to the successful positioning of body segments (elbow, hand, etc.) (Tarhan et al., 2015), often with respect to a static goal posture (Goldenberg, 1995; Goldenberg and Hagmann, 1997; Harrington and Haaland, 1997). However, for dynamic actions, the use of a body-configuration route may require linking together sequences of static postural configurations, whereas trajectory representations readily describe spatiotemporal information about motion along the desired path (e.g., instantaneous velocity). Interestingly, we saw a correlation between our task and a more conventional meaningless imitation task (Watson and Buxbaum, 2015), which reassures that there is likely a trajectory component in more conventional imitation tasks. Nevertheless, depending on the particular situation, imitation may be performed in two ways: by copying a movement trajectory or a bodypart-relationship goal. In support of this hypothesis, kinematic errors can dissociate from postural-configuration errors in imitation (Hermsdörfer et al., 1996).

Previously proposed neuroanatomical models of action planning have divided the dorsal stream (Ungerleider and Mishkin, 1982; Milner and Goodale, 1995) into two routes for action (Rizzolatti and Matelli, 2003; Binkofski and Buxbaum, 2013). Bodybased representations have been associated with the ventrodorsal pathway passing through the IPL (Goldenberg, 1995, 1999; Buxbaum et al., 2000; Schwoebel et al., 2004). In contrast, the dor- 
sodorsal stream (including PMd) has been associated with planning movements to targets in extrinsic space, and may thus represent actions in a body-independent manner. A bodyindependent trajectory representation for imitation could be supported by this latter pathway. Interestingly, our data also hint that performance on body-cued trials may be associated with more ventral brain regions, consistent with the dual-route model. Nevertheless, finding overlapping regions associated with bodycued and cursor-cued performance in both the Production and Perception tasks suggest a route from Perception to action specifically concerned with representing movement trajectories.

In conclusion, in this study, we tested the hypothesis, based on our previous research, that humans possess an ability to represent movement trajectories and that this representation is useful for imitating meaningless actions. We also sought to identify where in the praxis network spanning the left frontoparietal cortex such a trajectory-path representation exists. Our findings confirm the existence of an abstract (body-independent) trajectory-path representation for planning and executing complex actions, such as copying gestures, which may be dissociated behaviorally and neuroanatomically from the ability to accurately report the shape of the observed action. Furthermore, we provide strong evidence that damage to PMd disrupts this ability to represent trajectory shapes, giving rise to deficits in the ability to imitate meaningless actions.

\section{References}

Abend W, Bizzi E, Morasso P (1982) Human arm trajectory formation. Brain 105:331-348.

Albert NB, Ivry RB (2009) The persistence of spatial interference after extended training in a bimanual drawing task. Cortex 45:377-385.

Amorim MA, Isableu B, Jarraya M (2006) Embodied spatial transformations: "body analogy" for the mental rotation of objects. J Exp Psychol Gen 135:327-347.

Anderson JR, Bower GH (1972) Recognition and retrieval processes in free recall. Psychol Rev 79:97-123.

Bates D, Maechler M, Bolker B, Walker S (2015) Fitting linear mixed-effects models using lme4. J Stat Softw 67:1-48.

Bates E, Wilson SM, Saygin AP, Dick F, Sereno MI, Knight RT, Dronkers NF (2003) Voxel-based lesion-symptom mapping. Nat Neurosci 6:448450.

Bauer GB, Johnson CM (1994) Trained motor imitation by bottlenose dolphins (Tursiops truncatus). Percept Mot Skills 79:1307-1315.

Billard A, Epars Y, Calinon S, Schaal S, Cheng G (2004) Discovering optimal imitation strategies. Rob Auton Syst 47:69-77.

Binkofski F, Buxbaum LJ (2013) Two action systems in the human brain. Brain Lang 127:222-229.

Buxbaum LJ, Giovannetti T, Libon D (2000) The role of the dynamic body schema in praxis: evidence from primary progressive apraxia. Brain Cogn 44:166-191.

Buxbaum LJ, Johnson-Frey SH, Bartlett-Williams M (2005) Deficient internal models for planning hand-object interactions in apraxia. Neuropsychologia 43:917-929.

Buxbaum LJ, Shapiro AD, Coslett HB (2014) Critical brain regions for toolrelated and imitative actions: a componential analysis. Brain 137:19711985.

Carpenter RH (1981) Oculomotor procrastination. In: Eye movements: cognition and visual perception (Fisher DF, Monty RA, Senders JW, eds), pp 237-246. Hillsdale, NJ: Lawrence Erlbaum.

Caspers S, Zilles K, Laird AR, Eickhoff SB (2010) ALE meta-analysis of action observation and imitation in the human brain. Neuroimage 50: $1148-1167$.

Chaminade T, Meltzoff AN, Decety J (2005) An fMRI study of imitation: action representation and body schema. Neuropsychologia 43:115-127.

Cirstea MC, Mitnitski AB, Feldman AG, Levin MF (2003) Interjoint coordination dynamics during reaching in stroke. Exp Brain Res 151:289-300.

Cluff T, Scott SH (2015) Apparent and actual trajectory control depend on the behavioral context in upper limb motor tasks. J Neurosci 35: 12465-12476.
Davidoff J, Warrington EK (2001) A particular difficulty in discriminating between mirror images. Neuropsychologia 39:1022-1036.

DeMarco AT, Turkeltaub PE (2018) A multivariate lesion symptom mapping toolbox and examination of lesion-volume biases and correction methods in lesion-symptom mapping. Hum Brain Mapp 39:4169-4182.

Dewald JP, Sheshadri V, Dawson ML, Beer RF (2001) Upper-limb discoordination in hemiparetic stroke: implications for neurorehabilitation. Top Stroke Rehabil 8:1-12.

Flash T, Hogan N (1985) The coordination of arm movements: an experimentally confirmed mathematical model. J Neurosci 5:1688-1703.

Geschwind N (1975) The apraxias: neural mechanisms of disorders of learned movement. Am Sci 63:188-195.

Goldenberg G (1995) Imitating gestures and manipulating a mannikin: the representation of the human body in ideomotor apraxia. Neuropsychologia 33:63-72.

Goldenberg G (1999) Matching and imitation of hand and finger postures in patients with damage in the left or right hemispheres. Neuropsychologia 37:559-566.

Goldenberg G (2009) How the mind moves the body: lessons from apraxia. In: Oxford handbook of human action (Morsella E, Gollwitzer PM, eds). Oxford: Oxford UP.

Goldenberg G (2013) Apraxia: the cognitive side of motor control. Oxford: Oxford UP.

Goldenberg G, Hagmann S (1997) The meaning of meaningless gestures: a study of visuo-imitative apraxia. Neuropsychologia 35:333-341.

Goodall C (1991) Procrustes methods in the statistical-analysis of shape. J R Stat Soc Ser B 53:285-339.

Harrington DL, Haaland KY (1997) Representations of actions in ideomotor limb apraxia: clues from motor programming and control. In: Apraxia: the neuropsychology of action (Rothi LJ, Heilman KM, eds), pp 111 147. London: Psychology.

Hermsdörfer J, Mai N, Spatt J, Marquardt C, Veltkamp R, Goldenberg G (1996) Kinematic analysis of movement imitation in apraxia. Brain 119: $1575-1586$

Hocherman S, Wise SP (1990) Trajectory-selective neuronal activity in the motor cortex of rhesus monkeys (Macaca mulatta). Behav Neurosci 104: 495-499.

Hocherman S, Wise SP (1991) Effects of hand movement path on motor cortical activity in awake, behaving rhesus monkeys. Exp Brain Res 83: 285-302.

Hoeren M, Kümmerer D, Bormann T, Beume L, Ludwig VM, Vry MS, Mader I, Rijntjes M, Kaller CP, Weiller C (2014) Neural bases of imitation and pantomime in acute stroke patients: distinct streams for praxis. Brain 137:2796-2810

Hollingworth HL (1913) Characteristic differences between recall and recognition. Am J Psychol 24:532-544.

Ietswaart M, Carey DP, Della Sala S (2006) Tapping, grasping and aiming in ideomotor apraxia. Neuropsychologia 44:1175-1184.

Ijspeert AJ, Nakanishi J, Schaal S (2001) Trajectory formation for imitation with nonlinear dynamical systems. IEEE/RSJ International Conference on Intelligent Robots and Systems. Maui, HI.

Kadmon Harpaz N, Flash T, Dinstein I (2014) Scale-invariant movement encoding in the human motor system. Neuron 81:452-462.

Kalénine S, Buxbaum LJ, Coslett HB (2010) Critical brain regions for action recognition: lesion symptom mapping in left hemisphere stroke. Brain 133:3269-3280

Kalénine S, Shapiro AD, Buxbaum LJ (2013) Dissociations of action means and outcome processing in left-hemisphere stroke. Neuropsychologia 51: $1224-1233$

Kertesz A (1982) Western Aphasia Battery. New York: Grune \& Stratton.

Kintsch W (1970) Models for free recall and recognition. In: Models of human memory (Norman DA, ed), pp 331-373. San Diego: Academic.

Korneev AA, Kurgansky AV (2014) Internal representation of movement sequences on reproduction of static drawings and the trajectories of moving objects. Neurosci Behav Physiol 44:892-901.

Liepmann H (1905) The left hemisphere and action. London, Ontario: University of Western Ontario.

Mack L, Gonzalez Rothi LJ, Heilman KM (1993) Hemispheric-specialization for handwriting in right-handers. Brain Cognition 21:80-86.

Magnusson A, Skaug H, Nielsen A, Berg C, Kristensen K, Maechler M, van Bentham K, Bolker B, Brooks M (2017) glmmTMB: generalized linear mixed models using template model builder. R package version 013 . 
Mah YH, Husain M, Rees G, Nachev P (2014) Human brain lesion-deficit inference remapped. Brain 137:2522-2531.

Matarića MJ, Pomplunb M (1998) Fixation behavior in observation and imitation of human movement. Cogn Brain Res 7:191-202.

Milner AD, Goodale MA (1995) The visual brain in action. Oxford: Oxford UP.

Mirman D, Landrigan JF, Kokolis S, Verillo S, Ferrara C, Pustina D (2018) Corrections for multiple comparisons in voxel-based lesion-symptom mapping. Neuropsychologia 115:112-123.

Molenberghs P, Cunnington R, Mattingley JB (2009) Is the mirror neuron system involved in imitation? A short review and meta-analysis. Neurosci Biobehav Rev 33:975-980.

Moll L, Kuypers HG (1977) Premotor cortical ablations in monkeys: contralateral changes in visually guided reaching behavior. Science 198:317-319.

Moore BR (1992) Avian movement imitation and a new form of mimicry: tracing the evolution of a complex form of learning. Behaviour 122: 231-263.

Osu R, Ota K, Fujiwara T, Otaka Y, Kawato M, Liu M (2011) Quantifying the quality of hand movement in stroke patients through threedimensional curvature. J Neuroeng Rehabil 8:62.

Pearce TM, Moran DW (2012) Strategy-dependent encoding of planned arm movements in the dorsal premotor cortex. Science 337:984-988.

Penny WD, Friston KJ, Ashburner JT, Kiebel SJ, Nichols TE, eds (2007) Statistical parametric mapping: the analysis of functional brain images. London: Elsevier.

Petrides M, Milner B (1982) Deficits on subject-ordered tasks after frontaland temporal-lobe lesions in man. Neuropsychologia 20:249-262.

Pilacinski A, Wallscheid M, Lindner A (2018) Human posterior parietal and dorsal premotor cortex encode the visual properties of an upcoming action. PLoS One 13:e0198051.

Platz T, Mauritz KH (1995) Human motor planning, motor programming, and use of new task-relevant information with different apraxic syndromes. Eur J Neurosci 7:1536-1547.

Rijntjes M, Dettmers C, Büchel C, Kiebel S, Frackowiak RS, Weiller C (1999) A blueprint for movement: functional and anatomical representations in the human motor system. J Neurosci 19:8043-8048.

Rizzolatti G, Matelli M (2003) Two different streams form the dorsal visual system: anatomy and functions. Exp Brain Res 153:146-157.

Rumiati RI, Weiss PH, Tessari A, Assmus A, Zilles K, Herzog H, Fink GR (2005) Common and differential neural mechanisms supporting imitation of meaningful and meaningless actions. J Cogn Neurosci 17:14201431.

Schwoebel J, Buxbaum LJ, Coslett HB (2004) Representations of the human body in the production and imitation of complex movements. Cogn Neuropsychol 21:285-298.

Sergio LE, Scott SH (1998) Hand and joint paths during reaching movements with and without vision. Exp Brain Res 122:157-164.

Sperber C, Wiesen D, Karnath HO (2018) An empirical evaluation of multivariate lesion behaviour mapping using support vector regression. Hum Brain Mapp. Advance online publication. Retrieved December 13, 2018. doi: $10.1002 / \mathrm{hbm} .24476$.

Subiaul F (2016) What's special about human imitation? A comparison with enculturated apes. Behav Sci (Basel) 6:E13.

Tarhan LY, Watson CE, Buxbaum LJ (2015) Shared and distinct neuroanatomic regions critical for tool-related action production and recognition: evidence from 131 left-hemisphere stroke patients. J Cogn Neurosci 27:2491-2511.

Taylor CK, Saayman G (1973) Imitative behavior by Indian Ocean bottlenose dolphins (Tursiops aduncus) in captivity. Behavior 44:286-298.

Tseng Y, Scholz JP, Schöner G (2002) Goal-equivalent joint coordination in pointing: affect of vision and arm dominance. Motor Control 6:183-207.

Tulving E (1976) Ecphoric processes in recall and recognition. In: Recall and recognition (Brown J, ed), pp 37-74. London: Wiley.

Ungerleider LG, Mishkin M (1982) Two cortical visual systems. In: Analysis of visual behavior (Goodale MA, Ingle DJ, Mansfield RJ, eds), pp 549_ 586. Cambridge: Massachusetts Institute of Technology.

van Galen GP, Teulings HL (1983) The independent monitoring of form and scale factors in handwriting. Acta Psychol (Amst) 54:9-22.

Watson CE, Buxbaum LJ (2015) A distributed network critical for selecting among tool-directed actions. Cortex 65:65-82.

Weiss PH, Dohle C, Binkofski F, Schnitzler A, Freund HJ, Hefter H (2001) Motor impairment in patients with parietal lesions: disturbances of meaningless arm movement sequences. Neuropsychologia 39:397-405.

Whiten A, Horner V, Litchfield CA, Marshall-Pescini S (2004) How do apes ape? Learn Behav 32:36-52.

Wing AM (2000) Motor control: mechanisms of motor equivalence in handwriting. Curr Biol 10:R245-R248.

Wong AL, Goldsmith J, Krakauer JW (2016) A motor planning stage represents the shape of upcoming movement trajectories. J Neurophysiol 116:296-305.

Wright CE (1990) Generalized motor programs: reexamining claims of effector independence in writing. In: Attention and Performance XIII (Jeannerod M, ed), pp 294-320. Hillsdale, NJ: Lawrence Erlbaum.

Zhang Y, Kimberg DY, Coslett HB, Schwartz MF, Wang Z (2014) Multivariate lesion-symptom mapping using support vector regression. Hum Brain Mapp 35:5861-5876. 